\title{
INDIVIDUAL BANANA TREE CROWN DELINEATION USING UNMANNED AERIAL VEHICLE (UAV) IMAGES
}

\author{
Sudeep Kuikel ${ }^{1 *}$, Binita Upadhyay ${ }^{1}$, Dhruba Aryal ${ }^{1}$, Sudeep Bista ${ }^{1}$, Basant Awasthi ${ }^{1}$, Sanjeevan Shrestha ${ }^{2}$ \\ 1: Kathmandu University, Nepal; 2: Land Management Training Centre, Nepal
}

KEY WORDS: Tree Crown Delineation, Convolution Neural Network, Support Vector Machine, Object Based Image Analysis

\begin{abstract}
Individual Tree Crown (ITC) delineation from aerial imageries plays an important role in forestry management and precision farming. Several conventional as well as machine learning and deep learning algorithms have been recently used in ITC detection purpose. In this paper, we present Convolutional Neural Network (CNN) and Support Vector Machine (SVM) as the deep learning and machine learning algorithms along with conventional methods of classification such as Object Based Image Analysis (OBIA) and Nearest Neighborhood (NN) classification for banana tree delineation. The comparison was done based by considering two cases; Firstly, every single classifier was compared by feeding the image with height information to see the effect of height in banana tree delineation. Secondly, individual classifiers were compared quantitatively and qualitatively based on five metrices i.e., Overall Accuracy, Recall, Precision, F-Score, and Intersection Over Union (IoU) and best classifier was determined. The result shows that there are no significant differences in the metrices when height information was fed as there were banana tree of almost similar height in the farm. The result as discussed in quantitative and qualitative analysis showed that the CNN algorithm out performed SVM, OBIA and NN techniques for crown delineation in term of performance measures.
\end{abstract}

\section{INTRODUCTION}

Precision Agriculture is an innovative, integrated and internationally standardized approach aiming to increase the efficiency of resource used and reduce the uncertainty decisions required to manage variability on farms (Patali \& Chetan, 2016). Remote Sensing has been the precious gift in the field of precision agriculture (Melissari, 2016). Many works have been performed in calculating the parameters such as vegetation indices, crop height, crop yield, leaf area index, surface soil properties, ground biomass, water stress, canopy height models and many more (Zhang \& Kovacs, 2012). In the precision agriculture mainly in plantation industry and efficient forestry management, tree detection and delineation has become one of the important factors (Neupane, 2019). Data on individual trees are important inputs for biomass estimation, biodiversity assessments and forest growth models (Zaforemska, Xiao, \& Gaulton, 2020). Various research and projects have been carried out in tree detection and delineation for forestry and precision agriculture have been growing over the last few decades particularly adopting remote sensing (Syed, 2019). The rapid development of new algorithm has recently been parallel to the technological advances in the field of remote sensing.

The banana plant is a large perennial herb. The plant has $8-12$ leaves that are up to $270 \mathrm{~cm}$ long and $60 \mathrm{~cm}$ wide. The banana plants have star like shaped or mostly irregular. Bananas grow best in areas with $2,500 \mathrm{~mm}$ or more of well-distributed rainfall per year. Thailand being located in a tropical area; the growth and development of banana plants are affected due to different diseases. Despite of following all standards of planting, banana farms in Thailand still face a problem of losing plants within first few months of cultivation (Neupane, 2019). To keep track of productivity, counting the number of plants needs to be automated, rather than tedious manual approach.

Machine/Deep Learning (ML/DL) techniques have been applied to large data sets in order to extract relevant information and for making predictions. The performance and the outcomes of different ML/DL algorithms may vary depending upon the data sets being used, as well as on the suitability of algorithms to the data and the application domain under consideration (Awan,
Saleem, \& Crespi, 2020). Many researches have been already carried out for and delineation tree detection by using machine learning algorithm. SVM was used on Crop disease detection and classification of Banana Plant. But very few researches have been made on images generated by RGB cameras on board of unmanned aerial vehicle's (UAV) (Santos A. A., 2019). Deep learning has been highly used in the application of individual tree crown (ITC) detection in recent years. $\mathrm{CNN}$ is the standard approach for applications involving image classification, image processing, image recognition, object detection, etc. However, the convolution layers require huge amounts of processing time and resources (Neupane, 2019) . In this research, four different genres of classifier were used for Banana Tree Delineation. Among them, deep learning-based algorithms CNN shows the best performance and machine learning based algorithms SVM shows second best performance compared to other algorithms.

\section{STUDY AREA}

Huai Khwang is one of the 50 districts in Thailand. The temperature of this district is overcast in wet season whereas dry season will remain humid however; the average temperature throughout the year will be hot and warm. Over the course of time, the temperature will vary from $71^{\circ} \mathrm{F}$ to $95^{\circ} \mathrm{F}$ and is rarely below $65^{\circ} \mathrm{F}$ or above $99^{\circ} \mathrm{F}$. Bangkok is the favorable province for the banana plantation because of the fact that banana require as much warmth. Banana will grow in humid soils and requires well-drained field to thrive. This is one of the reasons that made us choose Bangkok as our project site. For our study we used the area of approximately 6.8 hectares of banana farm. Figure 1 . shows the study area for our project.

\section{METHODOLOGY}

The process of banana tree crown delineation includes various steps. We begin from planning, the required image was collected, the proposed algorithms were reviewed and explored, suitable performance metrices were selected for accuracy assessment and the final result was obtained. Figure 2 shows overall methodological process of banana tree crown delineation.

*Corresponding Author 


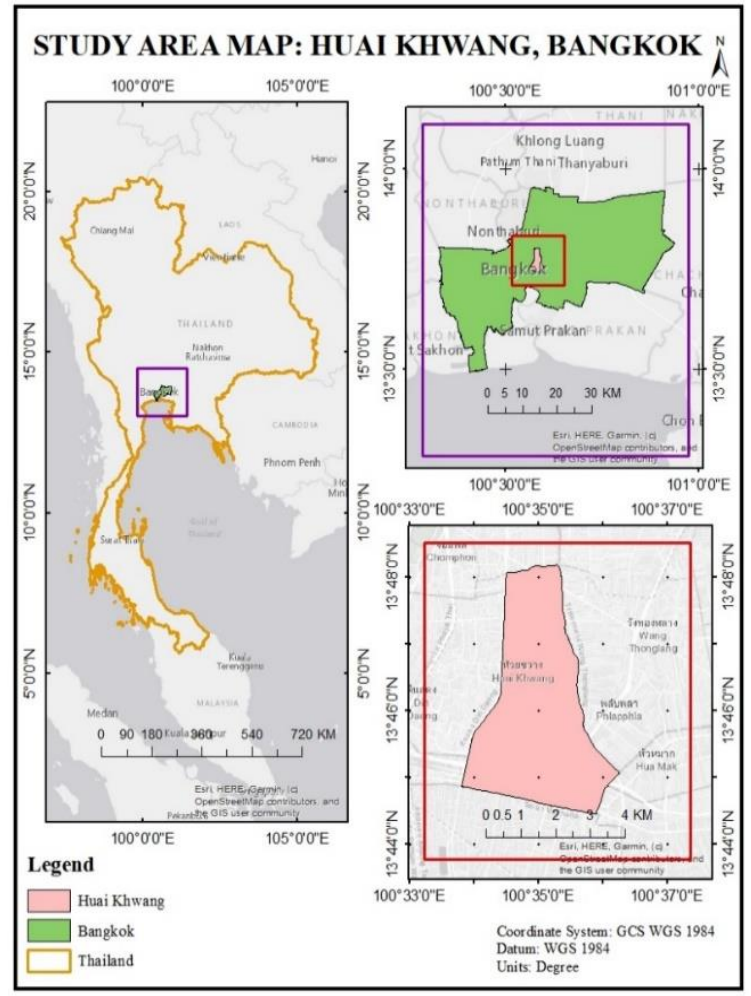

Figure 1. Study Area: Kings Fruits International Banana Farm

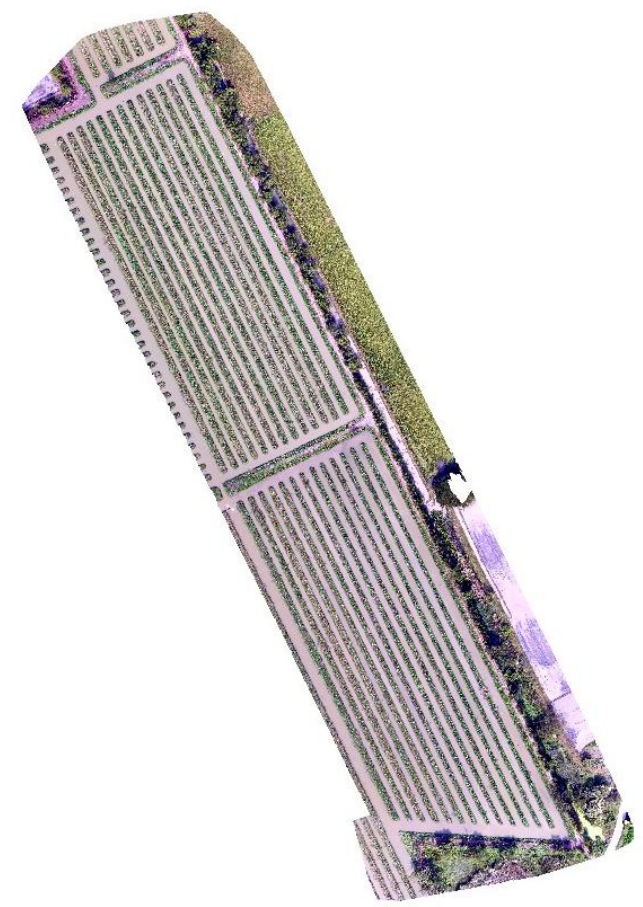

Figure 2. Orthophoto of Banana Farm

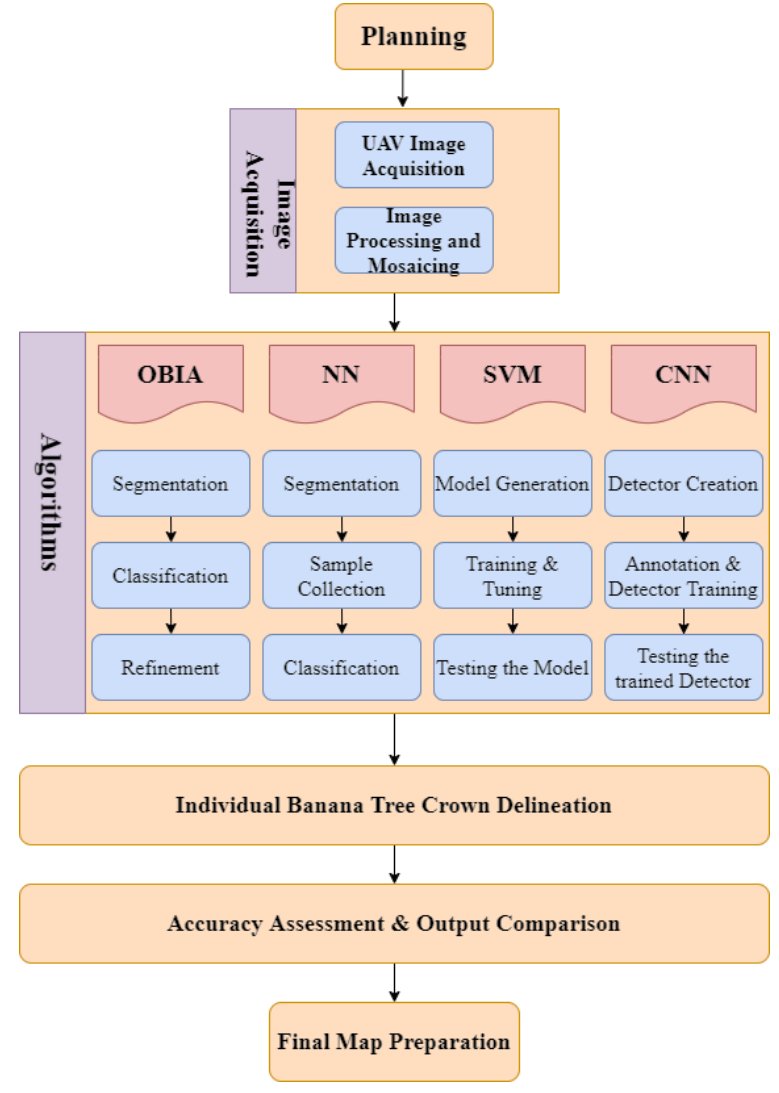

Figure 3. Methodological Flowchart of Banana Tree Crown Delineation

\subsection{Image Acquisition \& Processing}

We work on the UAV image collected by "Advance Geospatial Technology Research Unit, Sirindhorn International Institute of Technology, Thammasat University, Thailand who collected the Images over a commercial banana farm of Kings Fruits International Co., Ltd, Thailand. DJI Phantom 3 Drone was deployed for acquiring the images of the chosen banana farm using Pix4D Capture. The spatial resolution of the image was $1.7 \mathrm{~cm} /$ pixel and the flight height were $40 \mathrm{~m}$. Pix4D Mapper was used for the image processing which adjusts geometric and radiometric effects and help stitch the entire individual images and results in orthomosaic generation.

\subsection{Datasets Preparation}

The orthophoto (6.8 hectares approximately) shown in Figure 2 was obtained from the post processing technique and the $80 \%$ of the whole orthophoto were used for training data while $20 \%$ were used for testing data (Neupane, 2019). The training area was divided into smaller tiles of $600 * 600$ pixels. The size of each tiles was $10.12 \mathrm{~m} * 10.12 \mathrm{~m}$. The individual banana plants were then manually digitized for preparing the labelled datasets. The different shapefiles used to digitize the tiles of the banana farm were then merged into the single shapefile. The merged shapefile was rasterized. The parameters such as Coordinate System, extent, resolution etc. of original image and rasterized dataset were kept same. For labelling, the rasterized datasets were reclassified and labelled Banana Pixels as 1 and Non-Banana Pixels as 0.

\subsection{Banana Tree Crown Delineation}

Our research focuses on the crown delineation and performance comparison of the different classifiers for tree crown delineation. Four different genres of classifiers were selected and compared for banana tree crown delineation. Each classifier was used in 
two type of datasets i.e., dataset with height information and without height information.

Object Based Image Analysis was one of those classifiers used for tree detection and delineation methods (Ke \& Quackenbush, 2008). As discussed above, two analysis were done. Initially, the banana crown delineation was done without using height details. In this method, we solely used the orthophoto and prepared the ruleset for classification. However, for analysis to be done including height details, layer stacked image that contains the height information (i.e., orthophoto along with Normalized DSM) was used. The steps like segmentation, classification and refinement were to done for tree crown delineation (Johansen et al., 2014). Initially, Multiresolution segmentation was performed using parameters such as scale, shape and compactness were taken as 50, 0.1 and 0.5 respectively by trial-and-error method. After performing the multiresolution segmentation to create segments that delineate meaningful objects, classification was done using appropriate ruleset. During the classification process, the parameters such as Green Red Vegetation Indices (GRVI), Excess Green and Green Value were used to define the ruleset. GRVI was defined to be $0.16<\mathrm{GRVI}<0.2$. Green Value was set as $>150$. Finally, refinement was done by using Excess Green as the main parameter and defining the value as $<=80$ Excess Green $<160$. While using the height details, height was taken as the additional parameter by setting the threshold value less than $2.5 \mathrm{~m}$ for banana plants.

Nearest Neighborhood (NN) Classification was another classifier we used for tree crown delineation (Zhao et al., 2008). The steps like segmentation, sample collection and classification were done for crown delineation. Multiresolution segmentation was done similarly like in OBIA. The classifier was trained using all the banana plants of the training area and the trained model was applied to the testing area for classification. Both OBIA and NN classifications were performed by using eCognition software.

Machine learning based algorithm, SVM was another used for tree crown delineation by classification (Ray, 2007). At first, datasets were prepared for feeding as discussed in section 3.2. Then, the model was developed by using Python Script. The model was trained by fitting in the training area. SVC model was used in which the hyperparameters such as $\mathrm{C}$, kernel and degree were set as 1.0, radial basis function (rbf), 3 respectively. $\mathrm{C}$ and gamma are considered to be the most important parameters known as learning and regularization parameters for RBF Kernel (Pedregosa, F. and Varoquaux, G. and Gramfort, A. and Michel et al., 2011). These are the parameters that can impact on the classified result and the performance of the developed model. It was found that the value of $\mathrm{C}$ and gamma had noticeable impact in the computational time and the complexity of the model (Fraj, n.d.). So proper tuning of the parameters was done by trial-anderror method and the value of $\mathrm{C}$ and gamma were set as 1 and 'auto' respectively as these values were found appropriate in our context. Finally, the model was saved and later applied in the testing area for banana crown delineation.

Deep Learning based algorithm, CNN was also used for tree crown delineation. Deep learning-based platform, Picterra was used. Picterra's users can build and deploy unique actionable and ready to use deep-learning models (Picterra, 2020). Picterra Platform has two different modes i.e., Training Mode and Detection Mode. The training mode has many pre-trained models which can be used for object detection. We created two detectors named as 'Tree_delineation_withoutheight' for delineating banana tree without incorporating height information and another detector named as 'Tree_delineation_withheight' for delineating banana tree considering its height information. After detector creation, we prepared model by annotating training samples in the training image. At first, we drew training area and annotate the particular object, which we wished to detect in that training area in its actual shape. The prepared model was trained simply by using 'Train Detector' tool. Then, we drew testing area over which object would be annotated after model training. The trained detector was added to our actual testing image and the banana tree were detected.

\subsection{Performance Comparison}

The performance of these classifiers was compared by using five performance comparison metrices. They were Overall Accuracy, Precision, Recall, F1-Score and Intersection over Union.

\section{RESULT AND DISCUSSION}

We analyzed the performance of the classifier on two types of datasets i.e., on height integrated dataset and on without height integrated datasets. The height integrated dataset was created by stacking Digital Height Model (DHM) with orthophoto. Furthermore, we analyzed the obtained results in two different ways i.e., Quantitatively and Qualitatively.

In Quantitative Analysis, we compared the performance of the different classifier based on the performance metrices. The Table 1 presented below shows the performance of the different classifier.

Overall Accuracy is relative higher compared to other metrices because of the higher number of the non- banana pixels. Precision and Recall depends on the predicted number of the false positive and false negative. We cannot derive a conclusion for the large difference precision and recall values at such condition we prefer to use F- score. Intersection over Union calculates the overlaps of the object detected by the model with the ground truth bounding areas. Higher IoU indicates large overlaps of object detected by the models with ground truth datasets. From the Table. 1, we can conclude that CNN has outperformed other used algorithms in tree crown delineation.

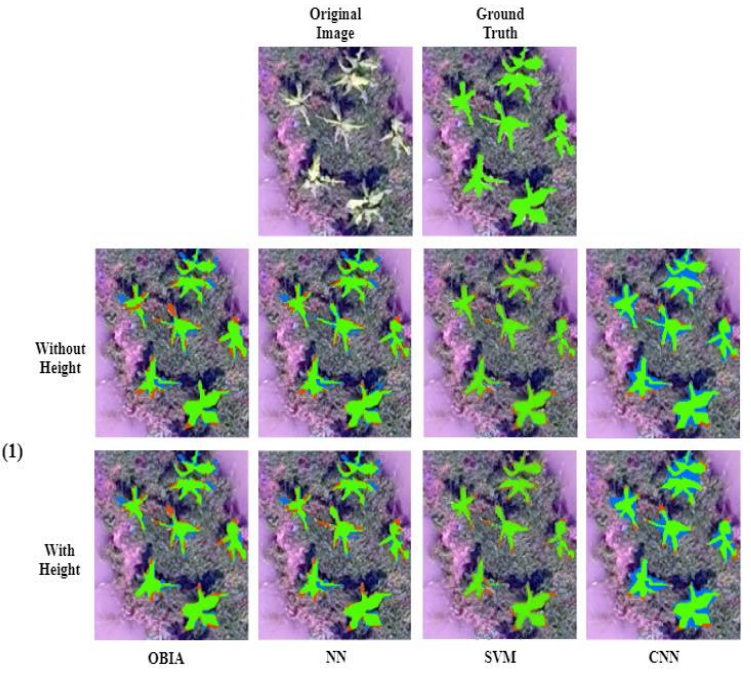

Figure 4. Visualization of prediction of four proposed model for Banana Tree Crown Delineation Image id (1) 
Table 1. Performances Metrices Values of Classifier

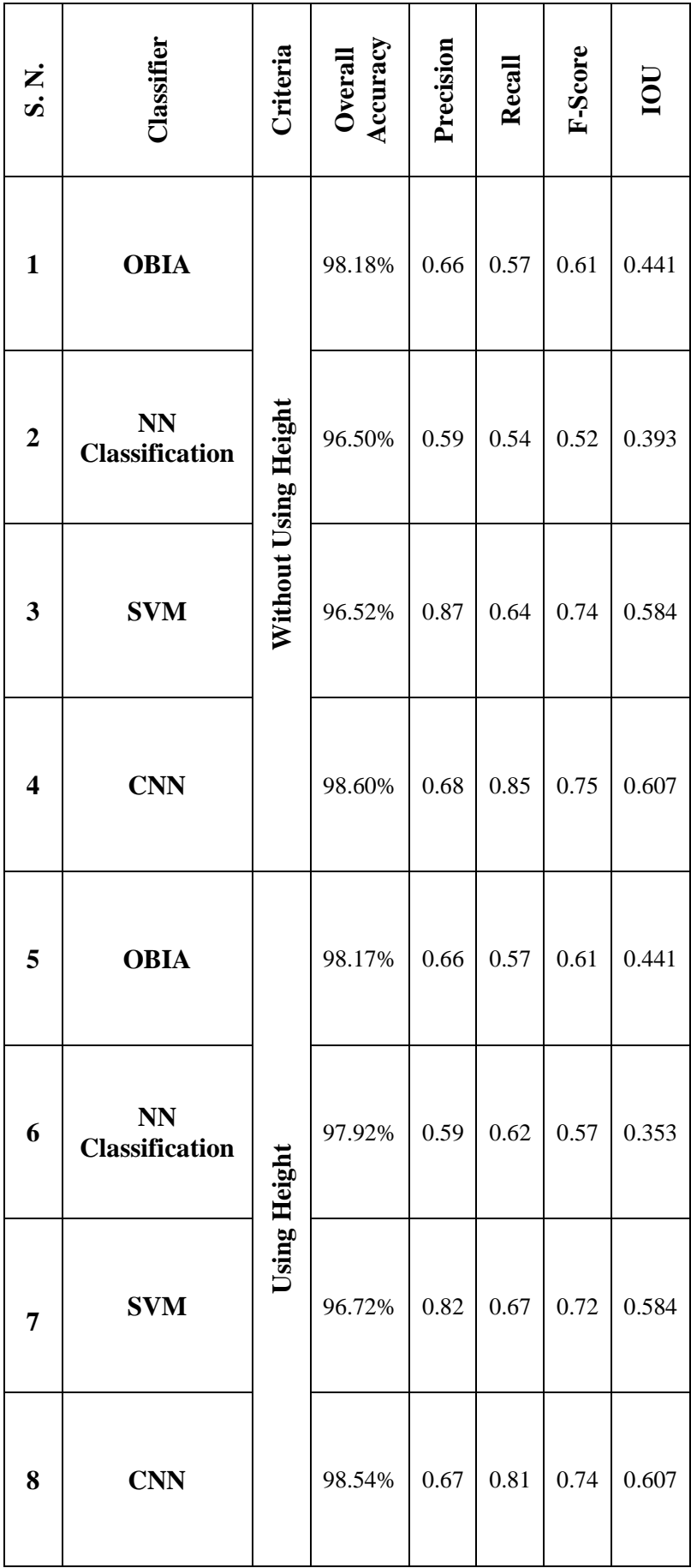

In Qualitative Analysis, we visually analyzed the performance of the classifier showing four terms of the confusion matrix i.e., True Positive, True Negative, False Positive and False Negative. We have selected four different areas from testing area which shows the performance variation of classifier.
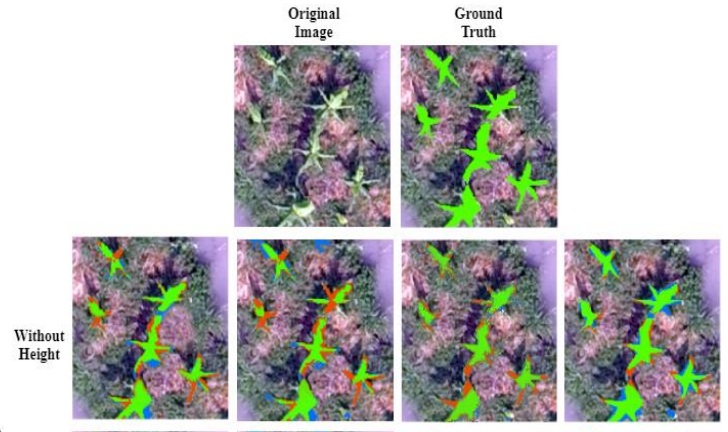

(2)
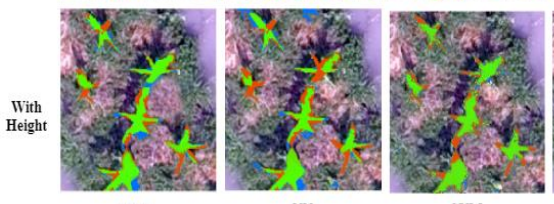

SVM

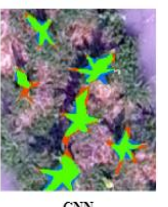

$\mathrm{CrN}$

Figure 5. Visualization of prediction of four proposed model for Banana Tree Crown Delineation. Image id (2)
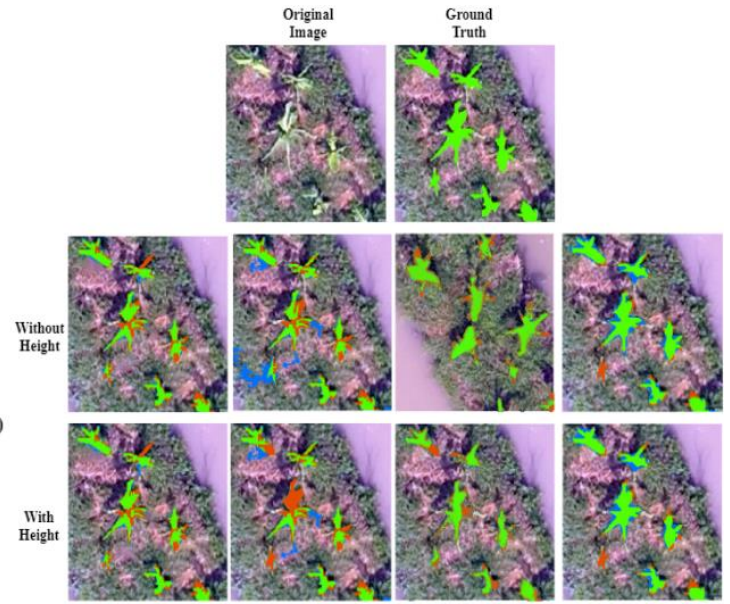

OBI
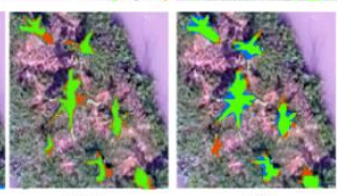

Figure 6. Visualization of prediction of four proposed model for Banana Tree Crown Delineation. Image id (3)
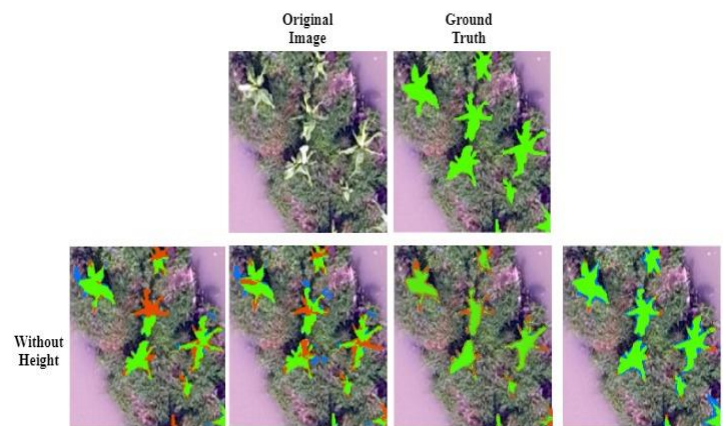

(3)

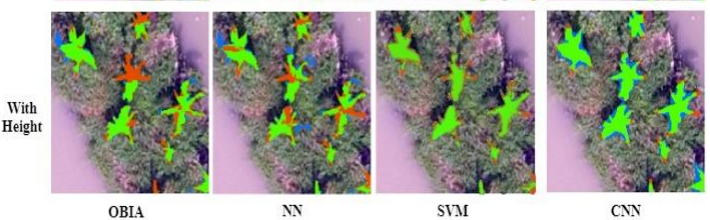

Figure 7. Visualization of prediction of four proposed model for Banana Tree Crown Delineation. Image id (4) 
In Figure 4, 5, 6 and 7 the first row shows the original image and the ground truth, second row shows performance of classifiers on without height integrated dataset and third row shows performance of classifiers on height integrated dataset. Green color pixel indicates true positive, blue color pixel indicates false positive, red color pixel indicates false negative and background represent true negative. This was visual inspection of the performance of the classifiers. The performance was best where the banana trees were distinct and equally spaced.

Table. 1 shows the values of the performance metrices for both type of datasets. We can observe that; height information hasn't significantly influenced the value of performance metrices compared to the dataset without height dataset. So, we concluded that height information didn't play important role in our case. It is because of the similar heights of banana plants in the farm.

From the Quantitative Analysis, we concluded that deep learning-based algorithm CNN outperformed largely in compared to conventional algorithm OBIA and NN but CNN slightly outperformed in comparison to machine learning algorithm SVM.

Similarly, Qualitative Analysis showed the result in accordance with quantitative analysis. It supports the conclusion of quantitative analysis because the performance metrices value obtained in quantitative analysis are highly correlated with the visually inspected result as discussed in qualitative analysis.

\section{CONCLUSION}

The main objective of the research conducted was to delineate the banana tree crown using UAV imageries exploiting different approaches (i.e., OBIA, NN classification, SVM and CNN). The performance of these methods was compared by feeding the algorithm with two types of datasets i.e., UAV images with height details and without height details. The result shows that height information didn't bring any significant changes in banana tree crown delineation as banana trees have no significant height difference. The quantitative analyses result shows less false positive in CNN and followed by SVM, OBIA and NN. This is also supported by high recall value of CNN (0.85), followed by SVM (0.64), OBIA (0.57) and NN (0.54) respectively. The major metrices i.e., F1-Score and IoU also indicated that $\mathrm{CNN}$ showed the best result for banana crown delineation correspond to the others proposed algorithms.

\section{REFERENCES}

Awan, F. M., Saleem, Y., \& Crespi, N. (2020). A comparative Analysis of Machine/Deep learning Models for Parking Space Availability Predictions. MDPI.

Fraj, M. Ben. (n.d.). In Depth: Parameter tuning for SVC $\mid$ by Mohtadi Ben Fraj | All things AI | Medium. Retrieved October 18, 2020, from https://medium.com/all-thingsai/in-depth-parameter-tuning-for-svc-758215394769

Johansen, K., Sohlbach, M., Sullivan, B., Stringer, S., Peasley, D., \& Phinn, S. (2014). Mapping banana plants from high spatial resolution orthophotos to facilitate plant health assessment. Remote Sensing, 6(9), 8261-8286. https://doi.org/10.3390/rs6098261

Ke, Y., \& Quackenbush, L. J. (2008). Comparison of individual tree crown detection and delineation methods. American Society for Photogrammetry and Remote Sensing ASPRS Annual Conference 2008 - Bridging the Horizons: New Frontiers in Geospatial Collaboration, 1 , $332-342$.
Melissari, B. (2016). Remote Sensing Technologies in Precise Framing. Researchgate.

Neupane, B. (2019). Deep learning based banana plant detection and counting using high-resolution redgreen-blue (RGB) images collected from unmanned aerial vehicle $(U A V)$. PLOS ONE.

Patali, B., \& Chetan, H. (2016). Role of Remote Sensing in Precision Agricultural. Researchgate.

Pedregosa, F. and Varoquaux, G. and Gramfort, A. and Michel, V., and Thirion, B. and Grisel, O. and Blondel, M. and Prettenhofer, P., \& and Weiss, R. and Dubourg, V. and Vanderplas, J. and Passos, A. and Cournapeau, D. and Brucher, M. and Perrot, M. and Duchesnay, E. (2011). sklearn.svm.SVC — scikit-learn 0.23.2 documentation. Journal of Machine Learning Research, 12, 2825--2830. https://scikit-

learn.org/stable/modules/generated/sklearn.svm.SVC.ht $\mathrm{ml}$

Picterra. (2020). Retrieved from picterra.ch: https://picterra.ch/about/\#: :text=Picterra\%2C\%20a $\% 20$ Swiss $\% 20$ company $\% 2 \mathrm{C} \% 20$ provides,to\%20use $\% 20$ deep $\% 2$ Dlearning\%20models

Ray, S. (2007). SVM | Support Vector Machine Algorithm in Machine Learning. https://www.analyticsvidhya.com/blog/2017/09/understai ng-support-vector-machine-example-code/

Santos, A. A. (2019). Assessment of CNN-Based Method for individual Tree Detection On image Captured by RGB Cameras Attached to UAVs. Sensors.

Syed, H. (2019). A report on Remote Sensing-based Method for Tree Detection and Delineation. IOP Conference Series: Material Science and Engineering.

Zaforemska, A., Xiao, W., \& Gaulton, R. (2020, Sept 21). Individual Tree Detection from UAV LIDAR Data ina a mixed species woodland. Retrieved from ISPRS Archieve: https://www.int-arch-photogramm-remotesens-spatial-inf-sci.net/XLII-2-W13/657/2019/

Zhang, C., \& Kovacs, J. M. (2012, july 21). The application of small unmanned aerial system for precision agriculture : a review. Retrieved from Link Springer: https://link.springer.com/article/10.1007/s11119-0129274-5

Zhao, F., Zhang, H., Li, Z., \& Pang, Y. (2008). The extraction of individual tree-crown in aerial digital camera imagery. Proceedings - 5th International Conference on Fuzzy Systems and Knowledge Discovery, FSKD 2008, 3, 183188. https://doi.org/10.1109/FSKD.2008.277 